\title{
Esophageal neuroendocrine tumor: A rare finding
}

\author{
Peter Stein, Stuart Akerman, Rachel Tannenbaum \\ Department of Gastroenterology, North Shore Long I sland Jewish Health System, New York, United States. \\ Correspondence: Peter Stein. Address: Department of Gastroenterology, North Shore Long Island Jewish Health \\ System, 300 Community Drive Manhasset, New York 11103, United States. Email: peterhstein@gmail.com \\ Received: January, 19, 2015 \\ DOI : $10.5430 /$ crim.v2n2p22 \\ Accepted: February 8, $2015 \quad$ Online Published: March 1, 2015 \\ URL: http://dx.doi.org/10.5430/crim.v2n2p22
}

\section{Abstract}

Neuroendocrine tumors of the esophagus are exceedingly rare, with few cases reported in the literature. Here, we present a patient with a complaint of dysphagia who was found to have this rare finding. Due to the lack of available data, little information exists regarding treatment for this disease. Our patient was successfully treated with combination chemotherapy and radiation with regression of disease on follow-up imaging. We seek to increase awareness of this exceedingly rare but dangerous disease presentation through sharing our unexpected finding.

\section{Keywords}

Neuroendocrine tumor, Endoscopic ultrasound

\section{Introduction}

Neuroendocrine tumors of the esophagus are quite rare, with few cases reported in the current literature. Here we highlight a case of a patient presenting with the relatively common symptom of mild dysphagia. This case illustrates how a seemingly mundane patient presentation can result in a truly rare and interesting underlying disease process. A review of the current literature shows the marked minority of neuroendocrine tumors presenting in the esophagus. Additionally, few patients have been reported to experience rapid and/or prolonged disease response to chemotherapy and radiation. Herein, we present an additional case and a review of the relevant literature.

\section{Case presentation}

A 76-year-old female presented with a 2-month history of mild intermittent dysphagia to solids only, with a few episodes of forced regurgitation. She initially lost 8 pounds thought to be secondary to her dysphagia, followed by a period of stabilization of her weight. Despite initial weight loss, her appetite remained intact. The patient had an EGD 2 years prior contributory only for gastric fundic gland polyps proven by biopsy. Her medical history was significant only for hyperlipidemia. The patient denied a history of tobacco, alcohol, or illicit drug use. On presentation, the patient's vital signs were stable, and physical examination was unremarkable for any pertinent findings. Laboratory testing including a CBC and CMP did not reveal any abnormalities.

An upper endoscopy was performed that revealed a friable non-obstructing lesion in the mid-esophagus $28 \mathrm{~cm}$ from the incisors. The lesion was well-circumscribed, approximately $3 \mathrm{~cm}$ in length, non-circumferential, and of the same color of 
surrounding normal esophageal mucosa (see Figure 1 \& 2). No evidence of Barrett's mucosa was present. Biopsy specimens were obtained showing a poorly differentiated neuroendocrine tumor. A subsequent CT scan of the chest was performed showing questionable thickening of the distal esophagus, and a peri-hepatic necrotic lymph node. No comments were made regarding any mid-esophageal abnormalities on CT. The patient subsequently underwent an esophageal EUS revealing a hypoechoic, irregular lesion 27-31 cm from the incisors. The mass extended through the submucosa but did not invade into the muscularis propria (T2 lesion). A $2.8 \mathrm{~cm} \times 2.3 \mathrm{~cm}$ round hypoechoic lymph node was identified in the celiac axis. No additional lesions were identified. The distal esophagus corresponding to the area of thickening on CT appeared unremarkable on EUS. FNA sampling with a 22-gauge core biopsy needle was performed of both the esophageal lesion and the celiac lymph node (see Figure 3, 4 \& 5). The mass was staged as T2N1.

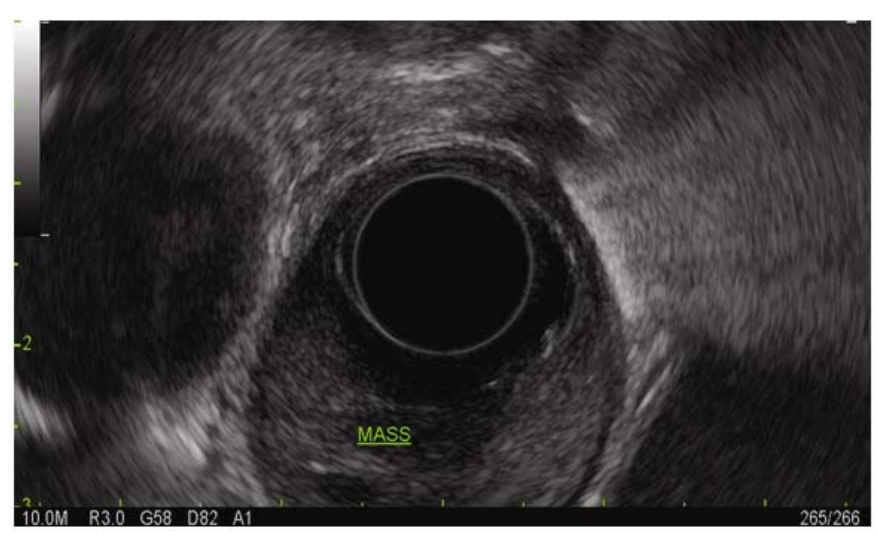

Figure 1. Mass at $28 \mathrm{~cm}$

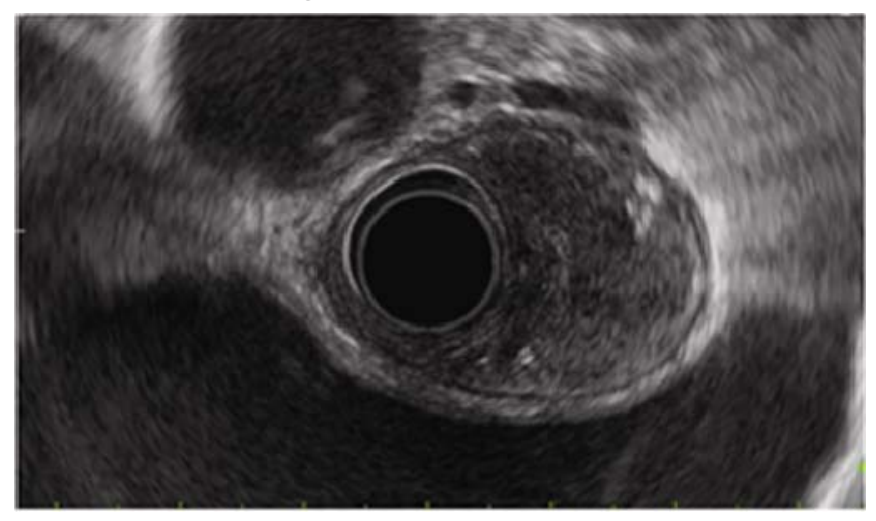

Figure 2. Mass at $29 \mathrm{~cm}$

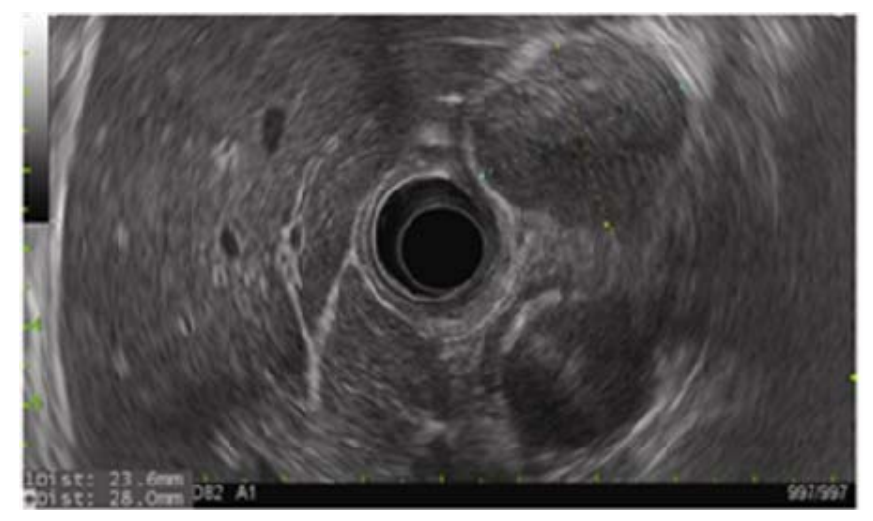

Figure 3. Celiac plexus lymph node with corresponding measurements 


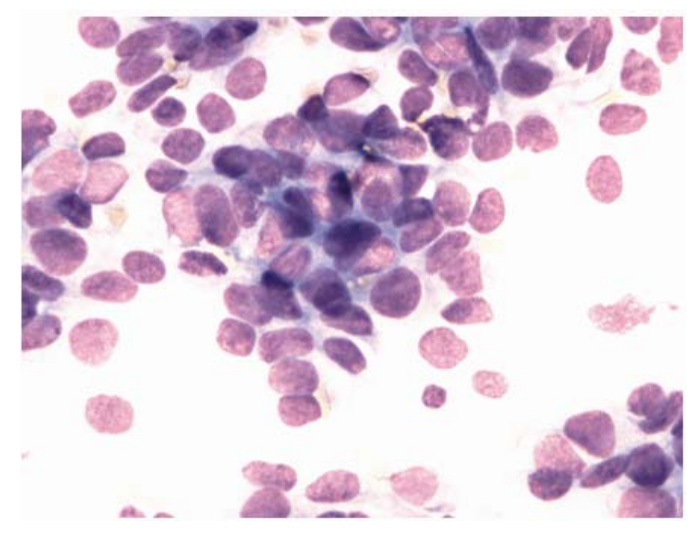

Figure 4. Cytology $\mathrm{H}$ and $\mathrm{E}$ staining

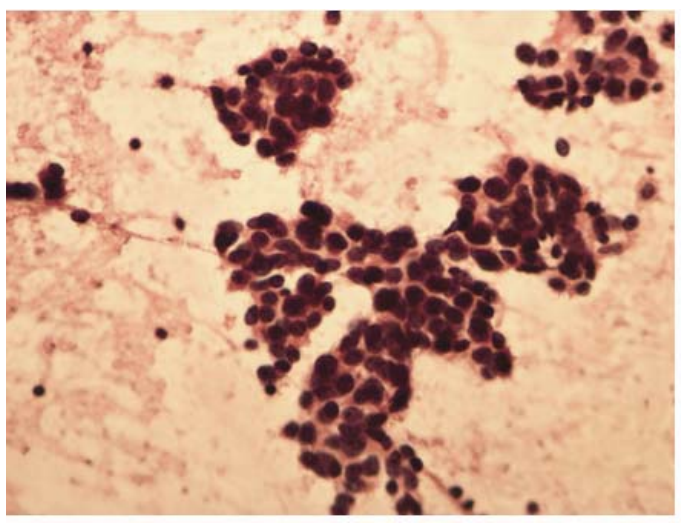

Figure 5. Core biopsy

Pathology results revealed a neuroendocrine carcinoma with squamous cell differentiation. CD56 staining was performed confirming chromogranin and synaptophysin positivity (see Figure 6). In addition, staining for CK56 was focally positive, and staining for S100, HMB45 was negative. A PET/CT was performed shortly after endoscopy showing increased activity corresponding to the known esophageal mass and the celiac lymph node. Additional activity was seen within the right hepatic lobe suspicious for metastatic disease. The final staging of the lesions was T2N1M1.

Our patient was successfully treated with a combined chemotherapy of etoposide and cisplatin for seven cycles, in addition to a radiation regimen, with successful regression. Repeat staging PET-CT imaging and repeat EUS and EGD after completion of chemotherapy were performed and showed complete tumor regression. The patient is currently asymptomatic 15 months after treatment.

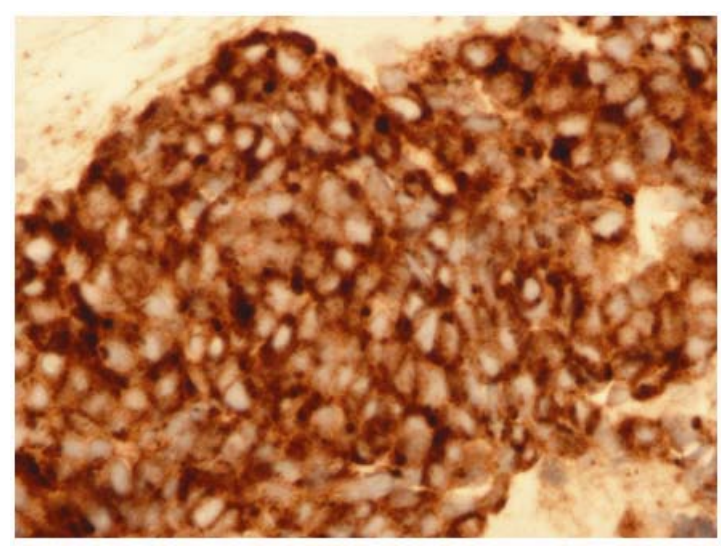

Figure 6. CD56 staining

\section{Discussion}

Neuroendocrine tumors of the esophagus are exceedingly rare, with few cases reported in the literature. In one study reporting on 8,305 neuroendocrine tumors at varying anatomical sites, only three were reported in the esophageus ${ }^{[1]}$. A separate Korean study reviewing 4,951 cases of gastroenteropancreatic neuroendocrine tumors identified only $1.4 \%$ within the esophagus ${ }^{[2]}$. A study in 2014 reviewing 2,037 pathology reports of gastroenteropancreatic neuroendocrine tumors identified 26 cases $(1.3 \%)$ in the esophagus ${ }^{[3]}$. To our knowledge, this is the first paper examining the clinical features and treatment options for patients with esophageal neuroendocrine tumors. Among the 26 patients identified in this recent study, $80.8 \%$ of patients were male with a mean age of 60 . Common presenting symptoms included dysphagia, 
abdominal discomfort, weight loss, and melena. Interestingly, only 1 patient studied out of 26 experienced hot flushes, a typical symptom of the carcinoid syndrome. Tumors were predominantly in the distal esophagus (76\%), and had a mean size of $2.34 \mathrm{~cm}$.

Our patient presented with dysphagia, the most common reported symptom. In addition, our patient presented with a regional lymph node suspicious for tumor spread. The location of this esophageal neuroendocrine tumor is not the only distinctive feature in our patient. The vast majority of lesions similar to that found in our patient occur in males. In addition, this classically slow-growing lesion was found after an unremarkable upper endoscopy two years prior. Our patient did not have any of the common presenting symptoms, other than mild intermittent dysphagia. This would be considered an uncommon presentation for an already metastatic rarely reported lesion.

Due to the lack of data, little information exists regarding treatment algorithms of esophageal neuroendocrine tumors. It has been postulated that tumors less than $1 \mathrm{~cm}$ in size without regional lymph node metastases can be safely resected endoscopically. Tumors larger than $1 \mathrm{~cm}$ should be surgically resected and should be treated with adjuvant chemotherapy. If regional lymph node metastases exist, radiotherapy would be indicated.

\section{References}

[1] Modlin IM, Sandor A. An analysis of 8305 cases of carcinoid tumors. Cancer. 1997; 79(4): 813-829. http://dx.doi.org/10.1002/(SICI)1097-0142(19970215)79:4<813::AID-CNCR19>3.0.CO;2-2

[2] Gastrointestinal Pathology Study Group of Korean Society of P, Cho MY, Kim JM, Sohn JH, et al. Current Trends of the Incidence and Pathological Diagnosis of Gastroenteropancreatic Neuroendocrine Tumors (GEP-NETs) in Korea 2000-2009: Multicenter Study. Canc Treat Res: Official Journal of the Korean Cancer Association. 2012; 44(3):157-165. PMid:23091441 http://dx.doi.org/10.4143/crt.2012.44.3.157

[3] Lee CG, Lim YJ, Park SJ, et al. The clinical features and treatment modality of esophageal neuroendocrine tumors: a multicenter study in Korea. BMC Cancer. 2014; (14): 569-576. PMid:25098730 http://dx.doi.org/10.1186/1471-2407-14-569 